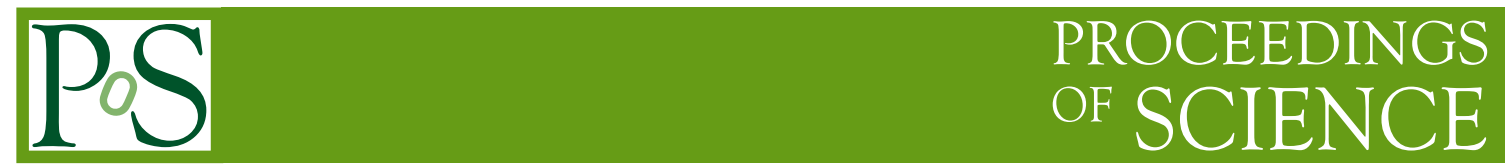

\title{
CP violation in $B_{s} \rightarrow J / \psi \phi:$ update from ATLAS
}

\section{Sinem Simsek on behalf of ATLAS Collaboration*}

Istanbul Bilgi University, Istanbul, Turkey

E-mail: sinem.sahin@cern.ch

Recent measurement of the time-dependent CP violating weak phase in $B_{s}^{0} \rightarrow J / \psi \phi$ decay using proton-proton collision data collected by the ATLAS detector at a center-of-mass energy of 13 $\mathrm{TeV}$ corresponding to integrated luminosity of $80.0 \mathrm{fb}^{-1}$ is presented. After combining the results with those from $19.2 \mathrm{fb}^{-1}$ of $7 \mathrm{TeV}$ and $8 \mathrm{TeV}$ data, the value of CP-violating phase is $\phi_{s}=-0.087$ \pm 0.036 (stat.) \pm 0.021 (syst.) rad.

BEAUTY2020

21-24 September 2020

Kashiwa, Japan (online)

${ }^{*}$ Speaker 


\section{Introduction}

The CP-violating weak phase, $\phi_{s}$, arises from the interference between the direct $b \rightarrow c \bar{c} s$ decay amplitude of $B_{s}$ and its decay amplitude via the $B_{s}-\overline{B_{S}}$ mixing. In the SM, the phase $\phi_{s}$ is small and related to the CP-violating phase of the $B_{s}$ unitarity triangle, $\beta_{s}=\arg \left[-\left(V_{t s} V_{t b}^{*}\right) /\left(V_{c s} V_{c b}^{*}\right)\right]$. Assuming no New Physics (NP) contributions to $B_{s}$ mixing and decays, the predictions of the weak phase that is made by the CKMFitter group [1] and the UTfit Collaboration [2] are $-2 \beta_{s}=$ $-0.03696_{-0.00082}^{+0.00072} \mathrm{rad}$ and $-2 \beta_{s}=-0.03700 \pm 0.00104 \mathrm{rad}$, respectively. Any deviation of the measured value from the Standart Model (SM) prediction could be a sign of the presence of the new physics processes.

Alongside $\phi_{s}$, the other quantities that describe the differential decay rate are the decay widths of the two mass eigenstates and the $\mathrm{CP}$ even/odd state amplitudes and phases.

We present a measurement of the $B_{s}^{0} \rightarrow J / \psi \phi$ decay parameters using $80.5 \mathrm{fb}^{-1}$ of the Large Hadron Collider (LHC) proton-proton (pp) data collected by the ATLAS detector during 2015-2017 at $13 \mathrm{TeV}$ center-of-mass energy [3]. The $80.5 \mathrm{fb}^{-1}$ based results were then combined with previous ATLAS results from Run1 [4] increasing the precision of physics parameters.

\section{ATLAS Experiment}

The ATLAS detector [5] is the largest, multi-purpose detector at the LHC. It was designed to investigate a wide range of physics including the particle physics beyond Standart Model (BSM). The detector has a cylindrical geometry and six different detecting subsystems wrapped concentrically in layers around the interaction point.

The inner detector (ID) covers the pseudorapidity range $|\eta|<2.5$, and consists of three main components: the silicon pixels (Pixel), the semiconductor tracker (SCT) and the transition radiation tracker (TRT). The tracking system, which is surrounded by a solenoid magnet with a magnetic field of 2T, is used to measure the track parameters of the charged particles. The next layers consist of a liquid-argon (LAr) sampling electromagnetic calorimeter and a hadronic calorimeter, which respectively provide good photon/electron identification, and precise jet and missing transverse energy measurements. The calorimeter system is followed by a muon spectrometer with three superconducting air-core toroids with a magnetic field of 4T, which are used to generate the magnetic field for the muon spectrometer (MS) to measure the magnetic deflection of the muon tracks.

\section{Reconstruction and Candidate Selection}

The basis of the trigger algorithms in order to select the events for the $B_{s}^{0} \rightarrow J / \psi \phi$ analysis is the identification of the $J / \psi \rightarrow \mu^{+} \mu^{-}$decays with the muon $p_{T}$ thresholds of either $4 \mathrm{GeV}$ or $6 \mathrm{GeV}$. In addition to the trigger requirement, the events must contain at least one reconstructed primary vertex, which was built from at least four inner detector tracks, and at least one pair of oppositely charged muon candidates that were reconstructed by combining the information from the ID and MS. Each muon pair is fitted to a common primary vertex, and accepted for the further analysis if the vertex fit satisfies the quality criteria $\chi^{2} /$ ndf $<10$. 
The approach to build the $\phi$ candidates is similar as for the $J / \psi$ candidates. All possible combinations of two oppositely charged tracks, which have not been previously identified as muons and fulfilled the requirements $p_{T}>1 \mathrm{GeV}$ and $|\eta|<2.5$, are selected for the analysis. The invariant mass of the two tracks is calculated under the assumption that they are kaons, and have to lie within the interval of $1.0085 \mathrm{GeV}<\mathrm{m}_{K^{+} K^{-}}<1.0305 \mathrm{GeV}$. In case of more than one candidate pass all the selections, the candidate with the lowest $\chi^{2} / \mathrm{ndf}$ is selected. In total, $2977576 B_{s}^{0}$ candidates have been collected within the mass range of $5.15-5.65 \mathrm{GeV}$.

For each $B_{s}^{0}$ meson candidate, the proper decay time, t, is estimated using:

$$
t=\frac{L_{x y} m_{B}}{p_{\mathrm{T}_{B}}}
$$

where $p_{\mathrm{T}_{B}}$ is the reconstructed transverse momentum of the $B_{s}^{0}$ meson candidate and $m_{B}$ denotes the mass of the $B_{s}^{0}$ meson, taken from Ref.[6]. The transverse decay length, $L_{x y}$, is the displacement in the transverse plane of the $B_{s}^{0}$ meson decay vertex with respect to the primary vertex, projected onto the direction of the $B_{S}^{0}$ transverse momentum.

\section{Flavour Tagging}

Determination of the initial flavour of the $B_{s}^{0}$ meson at the production point improves the measurement of the CP-violating phase $\phi_{s}$ in $B_{s}^{0} \rightarrow J / \psi \phi$ analysis. The information extracted from the decay chain on the opposite side of the $b-\bar{b}$ pair is used to infer the flavour of the initial B-hadron: this method is called opposite-side tagging (OST). In this analysis, identification of the initial flavour of the opposite B-hadrons is performed in the following tagging order: sets of two types of muons, sets of electrons, and b jets in the absence of a lepton in the opposite side decay. Each tagging method is based on the same discriminating variable, namely cone charge that is defined as follows:

$$
Q_{x}=\frac{\sum_{i}^{N \text { tracks }} q_{i} \cdot\left(p_{\mathrm{T} i}\right)^{\kappa}}{\sum_{i}^{N \text { tracks }}\left(p_{\mathrm{T} i}\right)^{\kappa}},
$$

where $x=\{\mu, e$, jet $\}$ refers to muon, electron, or b-jet charge, respectively.

The fact that there is no oscillation between the charged B mesons, unlike the neutral B mesons, makes it eaiser to indentify their production flavour from the decay products, which then can be used for the calibration of the OST algorithm by incorporating the yields of the signal $B_{s}^{0}$ mesons extracted from fits. For this aim, $B^{ \pm} \rightarrow J / \psi K^{ \pm}$candidate decays, where the kaon charge provides the information on the B meson charge, are used as the calibration sample. The invariant mass distribution for the selected $B^{ \pm}$candidates is shown in Figure 1 .

Once calibrated, the OST algorithms are applied to $B_{s}^{0} \rightarrow J / \psi \phi$ candidate events in order to obtain the probability whether each candidate is a $B_{s}^{0}$ or $\overline{B_{s}^{0}}$ meson state, which is then used in the maximum likelihood fit. Summary of tagging performances for the different flavour tagging methods is shown in Table 1. 


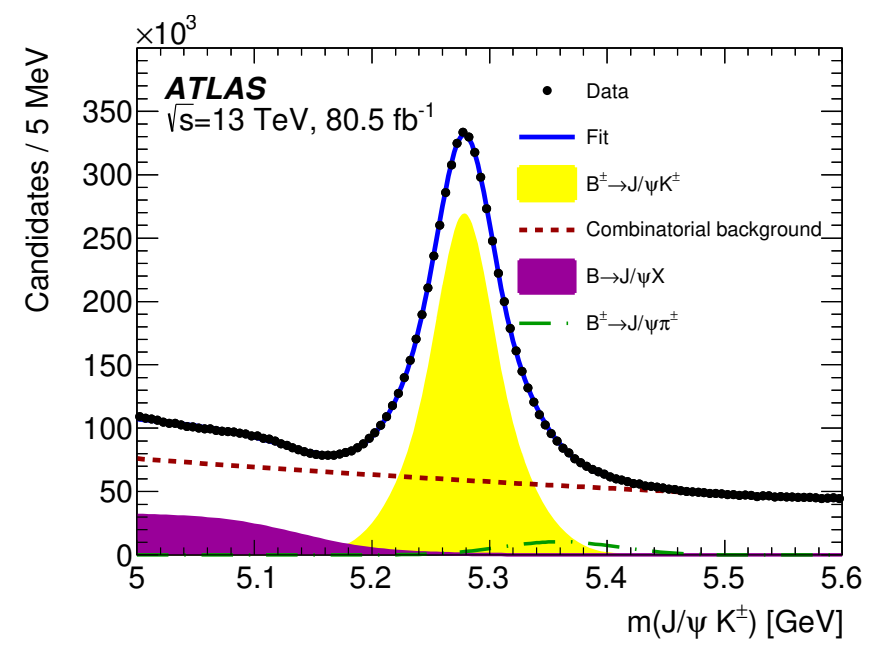

Figure 1: The invariant mass distribution for selected $B^{ \pm} \rightarrow J \psi K^{ \pm}$candidates.

Table 1: Summary of tagging performances for the different flavour tagging methods on the sample of $B^{ \pm}$ signal candidates.

\begin{tabular}{l|c|c|c}
\hline Tag method & $\epsilon_{x}[\%]$ & $D_{x}[\%]$ & $T_{x}[\%]$ \\
\hline \hline Tight muon & $4.50 \pm 0.01$ & $43.8 \pm 0.2$ & $0.862 \pm 0.009$ \\
Electron & $1.57 \pm 0.01$ & $41.8 \pm 0.2$ & $0.274 \pm 0.004$ \\
Low- $p_{T}$ muon & $3.12 \pm 0.01$ & $29.9 \pm 0.2$ & $0.278 \pm 0.006$ \\
Jet & $12.04 \pm 0.02$ & $16.6 \pm 0.1$ & $0.334 \pm 0.006$ \\
\hline Total & $21.23 \pm 0.03$ & $28.7 \pm 0.1$ & $1.75 \pm 0.01$ \\
\hline
\end{tabular}

\section{Maximum Likelihood Fit}

Since the $B_{s}^{0} \rightarrow J / \psi \phi$ decay is a decay of a pseudo-scalar into 2 vector particles, the final states are the admixture of CP-odd and CP-even states. In order to distinguish these states, a time depended angular analysis needs to be performed.

The decay amplitudes are decomposed using three independent linear polarization states $\left|A_{\|}(0)\right|^{2},\left|A_{0}(0)\right|^{2}$ and $\left|A_{S}(0)\right|^{2}$ of the vector mesons and are normalized such that $\left|A_{0}(0)\right|^{2}+$ $\left|A_{S}(0)\right|^{2}+\left|A_{\|}(0)\right|^{2}=1$. For each transversity amplitude, there is an associated phase $\delta_{0}=\arg \left(A_{0}\right)$ $\delta_{\perp}=\arg \left(\mathrm{A}_{\perp}\right)$ and $\delta_{\|}=\arg \left(\left|A_{\|}(0)\right|^{2}\right)$. However, the primary signal is diluted by other processes with the same final state such as non-resonant $B_{s}^{0} \rightarrow J / \psi K^{+} K^{-}$. These $\mathrm{S}$-wave states have been counted in the final description of the decay, using their own amplitude $\left|A_{S}(0)\right|^{2}$ and phase $\delta_{\|}$.

The analysis is based on the mass-lifetime angular fit, which is an unbinned maximum likelyhood fit to extract the physical parameters. The likelihood function is the combination of the signal and background probability density functions, and uses the information of the reconstructed mass, $\mathrm{m}$, the measured proper decay time, $\mathrm{t}$, the transversity angles of each candidate, $\Omega\left(\theta_{T}, \psi_{T}, \phi_{T}\right)$ and 
the tagging probability, $P\left(B \mid Q_{x}\right)$, as well as the other conditional observables such as the resolution of the mass, $\sigma_{m}$, and the resolution of the life time, $\sigma_{t}$, that are are constructed per canditate. The detailed explanation related to the likelihood function and the systematic uncertainties can be found in [3].

\section{Results}

The full simultaneous maximum likelihood fit contains nine physics parameters: $\Delta \Gamma_{s}, \phi_{s}, \Gamma_{s}$, $\left|A_{\|}(0)\right|^{2},\left|A_{0}(0)\right|^{2},\left|A_{S}(0)\right|^{2}, \delta_{\perp}, \delta_{\|}$and $\delta_{\perp}-\delta_{S}$. The number of signal $B_{s}^{0}$ meson candidates extracted from the fit is $453570 \pm 740$.

For most of the physics parameters including $\phi_{s}, \Delta \Gamma_{s}, \Gamma_{s}$, the fit determines a single solution while two well separated local maxima of the likelihood are found for the strong-phases $\delta_{\perp}$ and $\delta_{\|}$, which are shown as solution (a) and (b) in table of the results. The difference in the likelihoods, between the two solutions equals to 0.03 , favouring (a) but without ruling out (b). The results for the measured physics parameters of the simultaneous unbinned maximum likelihood fit are given in Table 2.

Table 2: Fitted values for the physics parameters of interest with their statistical and systematic uncertainties. The values for the variables $\delta_{\perp}$ and $\delta_{\|}$are denoted as solutions (a) and (b).

\begin{tabular}{c|c|c|c}
\hline Parameter & Value & $\begin{array}{c}\text { Statistical } \\
\text { uncertainty }\end{array}$ & $\begin{array}{c}\text { Systematic } \\
\text { uncertainty }\end{array}$ \\
\hline \hline$\phi_{s}[\mathrm{rad}]$ & -0.081 & 0.041 & 0.022 \\
$\Delta \Gamma_{s}\left[\mathrm{ps}^{-1}\right]$ & 0.0607 & 0.0047 & 0.0043 \\
$\Gamma_{s}\left[\mathrm{ps}^{-1}\right]$ & 0.6687 & 0.0015 & 0.0022 \\
$\left|A_{\|}(0)\right|^{2}$ & 0.2213 & 0.0019 & 0.0023 \\
$\left|A_{0}(0)\right|^{2}$ & 0.5131 & 0.0013 & 0.0038 \\
$\left|A_{S}(0)\right|^{2}$ & 0.0321 & 0.0033 & 0.04 \\
$\delta_{\perp}-\delta_{S}[\mathrm{rad}]$ & -0.25 & 0.05 & 1.2674 \\
\hline \multicolumn{4}{c}{ Solution (a) } \\
$\delta_{\perp}[\mathrm{rad}]$ & 3.12 & 0.11 & 0.06 \\
$\delta_{\|}[\mathrm{rad}]$ & 3.35 & 0.05 & 0.09 \\
\hline \multicolumn{3}{|c}{ Solution (b) } \\
$\delta_{\perp}[\mathrm{rad}]$ & 2.91 & 0.11 & 0.06 \\
$\delta_{\|}[\mathrm{rad}]$ & 2.94 & 0.05 & 0.09 \\
\hline
\end{tabular}

To combine the current results with those from the previous analysis, a best linear unbiased estimator (BLUE) is used. The measured values, uncertainties, and correlations are taken from the measurements performed at each centre-of-mass energy. The combined values of the physics parameters of $13 \mathrm{TeV}$ results with those obtained from $7 \mathrm{TeV}$ and $8 \mathrm{TeV}$ data can be seen in Table 3 . 
Table 3: The values of the physics parameters extracted in the combination of solution (a) and solution (b) of $13 \mathrm{TeV}$ results with those obtained from $7 \mathrm{TeV}$ and $8 \mathrm{TeV}$ data.

\begin{tabular}{c||c|c|c||c|c|c}
\hline \multicolumn{1}{c||}{ Parameter } & Value & $\begin{array}{c}\text { Statistical } \\
\text { uncertainty }\end{array}$ & $\begin{array}{c}\text { Systematic } \\
\text { uncertainty }\end{array}$ & Value & $\begin{array}{c}\text { Statistical } \\
\text { uncertainty }\end{array}$ & $\begin{array}{c}\text { Systematic } \\
\text { uncertainty }\end{array}$ \\
\hline \hline$\phi_{S}[\mathrm{rad}]$ & -0.087 & 0.036 & 0.021 & -0.087 & 0.036 & 0.021 \\
$\Delta \Gamma_{S}\left[\mathrm{ps}^{-1}\right]$ & 0.0657 & 0.0043 & 0.0037 & 0.0657 & 0.0043 & 0.0037 \\
$\Gamma_{S}\left[\mathrm{ps}^{-1}\right]$ & 0.6703 & 0.0014 & 0.0018 & 0.6704 & 0.0014 & 0.0018 \\
$\left|A_{\|}(0)\right|^{2}$ & 0.2220 & 0.0017 & 0.0021 & 0.2218 & 0.0017 & 0.0021 \\
$\left|A_{0}(0)\right|^{2}$ & 0.5152 & 0.0012 & 0.0034 & 0.5152 & 0.0012 & 0.0034 \\
$\left|A_{S}\right|^{2}$ & 0.0343 & 0.0031 & 0.0045 & 0.0348 & 0.0031 & 0.0045 \\
$\delta_{\perp}[\mathrm{rad}]$ & 3.22 & 0.10 & 0.05 & 3.03 & 0.10 & 0.05 \\
$\delta_{\|}[\mathrm{rad}]$ & 3.36 & 0.05 & 0.09 & 2.95 & 0.05 & 0.09 \\
$\delta_{\perp}-\delta_{S}[\mathrm{rad}]$ & -0.24 & 0.05 & 0.04 & -0.24 & 0.05 & 0.04 \\
\hline
\end{tabular}

\section{Summary}

This analysis presents a measurement of the parameters of the time-dependent CP asymmetry in $B_{s}^{0} \rightarrow J / \psi \phi$ decay from an $80.5 \mathrm{fb}^{-1}$ data sample of pp collisions collected with the ATLAS detector during the $13 \mathrm{TeV}$ LHC run. The results of this measurement in combination with $7 \mathrm{TeV}$ and $8 \mathrm{TeV}$ data are: $\phi_{s}=-0.087 \pm 0.036$ (stat.) \pm 0.021 (syst.) rad, $\Delta \Gamma_{s}=0.0657 \pm 0.0043$ (stat.) \pm 0.0037 (syst.) $\mathrm{ps}^{-1}$ and $\Gamma_{s}=0.6703 \pm 0.0014$ (stat.) \pm 0.0018 (syst.) $\mathrm{ps}^{-1}$.

These results are consistent with those obtained in the previous ATLAS analysis using $7 \mathrm{TeV}$ and $8 \mathrm{TeV}$ data. The measurement of the CP-violating phase $\phi_{s}$ is consistent also with the SM prediction with an improvement of the precision on the previous ATLAS results.

\section{References}

[1] CKMfitter group, Charles, J. et al., Current status of the Standard Model CKM fit and constraints on $\Delta F=2$ New Physics, Phys.Rev. D91 (2015) 073007.

[2] UTfit Collaboration, M. Bona et al., The unitarity triangle fit in the standard model and hadronic parameters from lattice QCD: A reappraisal after the measurements of $\Delta m_{s}$ and $B R(B \rightarrow \tau v \tau)$, JHEP 10 (2006) 081.

[3] ATLAS Collaboration, Measurement of the CP violation phase $\phi_{s}$ in $B_{s}^{0} \rightarrow J / \psi \phi$ decay in ATLAS at 13TeV, arXiv:2001.07115 [hep-ex], Accepted by EPJC.

[4] ATLAS Collaboration, Measurement of the CP-violating phase $\phi_{s}$ and the $B_{s}^{0}$ meson decay width difference with $B_{s}^{0} \rightarrow J / \psi \phi$ decays in ATLAS, JHEP 08 (2016) 147, arXiv:1601.03297 [hep-ex].

[5] ATLAS Collaboration, The ATLAS Experiment at the CERN Large Hadron Collider, JINST 3 (2008) S08003.

[6] M.Tanabashi et al., Review of Particle Physics, Phys. Rev. D98 (2018) 030001. 